\title{
The Human Retrosplenial Cortex and Thalamus Code Head Direction in a Global Reference Frame
}

\author{
Jonathan P. Shine, ${ }^{1}$ José P. Valdés-Herrera, ${ }^{1}$ Mary Hegarty, ${ }^{2}$ and Thomas Wolbers ${ }^{1,3}$ \\ ${ }^{1}$ German Center for Neurodegenerative Diseases (DZNE), Aging and Cognition Research Group, 39120 Magdeburg, Germany, ${ }^{2}$ Department of Psychological \\ and Brain Sciences, University of California, Santa Barbara, California, 93106, and ${ }^{3}$ Center for Behavioral Brain Sciences, 39118 Magdeburg, Germany
}

Spatial navigation is a multisensory process involving integration of visual and body-based cues. In rodents, head direction (HD) cells, which are most abundant in the thalamus, integrate these cues to code facing direction. Human fMRI studies examining HD coding in virtual environments (VE) have reported effects in retrosplenial complex and (pre-)subiculum, but not the thalamus. Furthermore, HD coding appeared insensitive to global landmarks. These tasks, however, provided only visual cues for orientation, and attending to global landmarks did not benefit task performance. In the present study, participants explored a VE comprising four separate locales, surrounded by four global landmarks. To provide body-based cues, participants wore a head-mounted display so that physical rotations changed facing direction in the VE. During subsequent MRI scanning, subjects saw stationary views of the environment and judged whether their orientation was the same as in the preceding trial. Parameter estimates extracted from retrosplenial cortex and the thalamus revealed significantly reduced BOLD responses when HD was repeated. Moreover, consistent with rodent findings, the signal did not continue to adapt over repetitions of the same HD. These results were supported by a whole-brain analysis showing additional repetition suppression in the precuneus. Together, our findings suggest that: (1) consistent with the rodent literature, the human thalamus may integrate visual and body-based, orientation cues; (2) global reference frame cues can be used to integrate HD across separate individual locales; and (3) immersive training procedures providing full body-based cues may help to elucidate the neural mechanisms supporting spatial navigation.

Key words: fMRI; head direction; human; navigation; retrosplenial cortex; thalamus

Significance Statement

In rodents, head direction (HD) cells signal facing direction in the environment via increased firing when the animal assumes a certain orientation. Distinct brain regions, the retrosplenial cortex (RSC) and thalamus, code for visual and vestibular cues of orientation, respectively. Putative HD signals have been observed in human RSC but not the thalamus, potentially because body-based cues were not provided. Here, participants encoded HD in a novel virtual environment while wearing a head-mounted display to provide body-based cues for orientation. In subsequent fMRI scanning, we found evidence of an HD signal in RSC, thalamus, and precuneus. These findings harmonize rodent and human data, and suggest that immersive training procedures provide a viable way to examine the neural basis of navigation.

\section{Introduction}

Head direction (HD) cells, examined extensively in rodents, code orientation information by firing when the animal assumes a

\footnotetext{
Received April 2, 2015; revised March 30, 2016; accepted April 21, 2016.

Author contributions: M.H. and T.W. designed research; T.W. J.P.S, and J.P.V.-H performed research; J.P.S and T.W. wrote the paper.

This work was supported by a Karp Discovery Award and by a Starting Grant of the European Research Council to T.W. (AGESPACE 335090). We thank Jack Loomis for helpful comments on an earlier version of this paper, and Masaki Miyanohara and Rebecca Korn for help with data collection.

The authors declare no competing financial interests.

Correspondence should be addressed to Dr Jonathan Shine, German Center for Neurodegenerative Diseases (DZNE), Aging and Cognition Research Group, Building 64, Room 519, Leipziger Stra $\beta$ e 44, 39120 Magdeburg, Germany. E-mail: Jonathan.shine@DZNE.de.
}

particular facing direction (for review, see Taube, 2007). These cells have been found in a number of regions, including retrosplenial cortex (RSC) and anterior dorsal thalamic nuclei (ADN). Although human functional imaging studies have reported HD coding in retrosplenial complex, a more extensive region of posteromedial cortex not limited to anatomical RSC (Baumann and Mattingley, 2010; Marchette et al., 2014), none have observed a contribution of the thalamus to this signal.

In rodents, HD cells increase in firing rate when the animal assumes a particular orientation. When the head is kept stationary, however, HD firing rates are maximal for the first $100 \mathrm{~ms}$ 
before stabilizing over several seconds to less than one-half of their initial firing rates (Shinder and Taube, 2014). The preferred firing direction of these cells is determined by both external visual, and internally generated, body-based, cues. Under normal conditions, the preferred firing direction of HD cells is governed strongly by visible landmarks (Taube et al., 1990a,b). Lesioning the RSC (Clark et al., 2010) or postsubiculum (PoS; Goodridge and Taube, 1997) reduces the influence of landmark position on HD cell firing, suggesting that these regions code visual orientation cues. ADN HD cells remain sensitive to facing direction, however, through self-motion cues. In contrast, removal of vestibular input completely disrupts orientation coding in the ADN, even in the presence of intact RSC and PoS (Stackman and Taube, 1997), suggesting that idiothetic information is critical for HD coding in this region. Accordingly, a key role of the ADN may be to integrate body-based and visual orientation cues (Clark et al., 2010).

Human fMRI studies examining HD coding in virtual environments (VE) have used visual, but not body-based, cues during training to differentiate orientation. Accordingly, effects observed in RSC (Baumann and Mattingley, 2010; Marchette et al., 2014), and subiculum (Chadwick et al., 2015) may reflect coding of visual cues for facing direction. Bodybased cues, such as proprioceptive feedback or motor efference copies, provide important information regarding one's heading in the environment, and can support navigation in the absence of visual input (eg, in path integration; Etienne and Jeffery, 2004). A task in which both body-based and visual information can be used to differentiate orientation, therefore, may be more likely to recruit the thalamus.

A second open question pertains to the reference frame in which HD is coded. Marchette et al. (2014) found that whereas RSC coded for HD in reference frames anchored to local environmental features, there was no evidence for HD coding in a global reference frame. The task demands may explain this effect because participants made directional judgments regarding proximal objects, rather than global landmarks, which may have promoted the use of local reference frames. Successfully navigating complex real world environments, however, requires the integration of disparate locales into a cohesive cognitive map (Wolbers and Wiener, 2014), which would benefit from orientation coding in a global reference frame (Vass and Epstein, 2013).

In the current experiment, we asked whether there was evidence of thalamic HD computations when body-based cues were provided during learning of an environment, and whether HD can be coded in a global reference frame. To achieve this, participants learned a VE comprising four distinct buildings while wearing a head-mounted display, requiring them to physically turn around to change orientation. In a subsequent one-back task performed during fMRI scanning, stimuli were presented that cued different HDs. We predicted adaptation in the RSC and the b

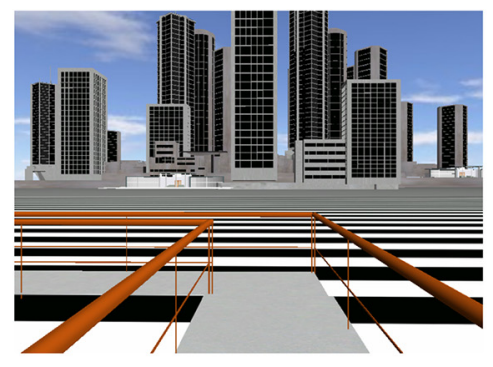

d

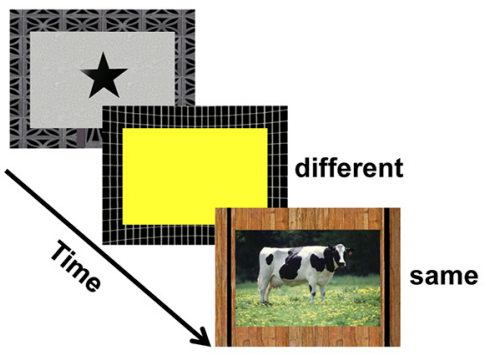

f

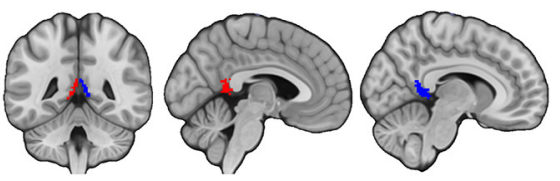

Figure 1. The VE environment in which participants learned individual images associated with different HDs, and an example (d) space and used to extract average percentage signal change values for the different repetition conditions.

thalamus, associated with repeating HD across buildings. Moreover, we analyzed the BOLD response over multiple repetitions of the same HD to test whether this signal plateaus, as has been demonstrated in rodents.

\section{Materials and Methods}

\section{Participants}

Nine healthy volunteers (2 female; age range: 19-32), all right-handed, with normal or corrected-to-normal vision, and no history of psychiatric disorder, participated in the experiment at the UC Santa Barbara Brain Imaging Center. The study received ethical approval from the local ethics committee.

\section{Stimuli}

A novel VE was created using 3D Studio Max comprising four unique, separate, enclosed rooms or "galleries" (2 square galleries, 1 circular, and 1 hexagonal) linked by a handrail (Fig. 1a). Four global landmarks (a cityscape, a bridge, and 2 different colored mountain ranges; Fig. 1b) were positioned at the cardinal $\operatorname{HDs}\left(0^{\circ}, 90^{\circ}, 180^{\circ}\right.$, and $\left.270^{\circ}\right)$, and the environment had a black and white striped floor. Each gallery housed a unique category of artwork (geometric shapes, abstract art, color blocks, or animals), and contained four unique paintings ( 16 in total in the VE). To further disambiguate the galleries, each one contained a unique wall texture (Fig. 1c). Within each gallery, the four paintings were arranged so that each one occupied a position concordant with a global landmark, effectively meaning that individual paintings occupied a specific HD.

For the one-back test phase administered during MRI scanning, individual paintings were displayed with a partial view of the background wall texture (Fig. 1d). 


\section{Procedure}

Training phase. The experiment comprised training and scanned test phases. During training, participants familiarized themselves with the $\mathrm{VE}$, and learned the specific paintings associated with different HDs, in advance of the one-back task in the scanner. To provide an immersive learning experience and to provide full body-based cues about selfrotation, participants wore a head-mounted display. The VE was projected to this display with motion trackers detecting head only or head and body rotations, which then provided an updated visual input so that the participant experienced full $360^{\circ}$ exploration within the VE. In contrast to rotational movements, forward/backward translational movements within the VE were produced solely by forward and backward motions of a joystick (ie, no translations of the body). Before exploration of the VE, participants were made aware that they would be tested on their knowledge of the paintings' orientations in the galleries relative to the global landmarks. They were advised to use all environmental cues available (ie, the orientation of the black and white striped floor, and the relative positions of the four global landmarks) to monitor their orientation while transitioning between, and entering, the galleries. Critically, once inside a gallery, participants had no visual access to the outside world. After entering a gallery, they were transported automatically to a marker in the center of the room (Fig. 1c). During this time, the participant's movement was confined to rotating in place to view the four items on the walls. Participants pressed a button to indicate when they had finished studying the paintings, at which point the pictures were removed and two doors appeared so that they could exit the room. It is important to note that, because of the layout of the environment, the egocentric bearings of the outside landmarks differed in each gallery. For example, across the four galleries, the cityscape was located egocentrically to the left, right, front, and behind the participant. Participants were required, therefore, to develop allocentric knowledge of HD in the VE.

After each circuit of the environment, participants were tested on the knowledge of their facing direction relative to the global cues when facing each of the 16 paintings. Participants were seated in front of a computer with a joystick, and presented with one of the paintings with a text prompt beneath it asking them to point in the direction of a specific global landmark. If the participant made a mean average pointing error $>10^{\circ}$ across trials, they were returned to the VE for a further training phase. Training continued until the participant reached criterion before moving onto the test phase, which was the fMRI component of the task.

Test phase. After reaching criterion in the training phase (ie, $<10^{\circ}$ mean average pointing error), participants performed a one-back task while undergoing MR scanning. In this task, the 16 paintings were presented individually, and participants were required to recall their facing direction associated with the painting in the current trial and compare it to their facing direction associated with the painting in the preceding trial. Given that participants were required to remain motionless in the scanner, it was our assumption that the visual cues would lead to a recapitulation of the information encoded with them during learning in the VE (ie, an encoding specificity effect; Tulving and Thomson, 1973; for review, see Watrous and Ekstrom, 2014). Several models of memory propose that associated event details are reinstated during retrieval of an episode (Norman and O'Reilly, 2003), and evidence for this cortical reinstatement has been demonstrated via stimulus-specific patterns of activity in fMRI (Johnson and Rugg, 2007), and electroencephalography (Wimberet al., 2012). In the current study, therefore, we assumed that the presentation of a visual cue to a specific HD would lead to reinstatement of the body-based cues about facing direction that were available during learning. Participants pressed one button if the facing directions differed over consecutive trials (non-repeat HD), and an alternate button if they were identical (repeat HD). Items were presented for $5 \mathrm{~s}$, and participants were told to respond as quickly and accurately as possible during this time. Trial order was controlled so that for both non-repeat, and repeat HDs, images were drawn from a different gallery (ie, a different category of stimuli) on each trial. Any effects associated with the one-back, therefore, could not be explained by repetition of a semantic/ visual category. A block comprised 32 trials, with each of the 16 items presented twice, serving once as a non-repeat HD trial, and once as a repeat $\mathrm{HD}$ trial (ie, 16 non-repeat $\mathrm{HD}$, and 16 repeat $\mathrm{HD}$ trials per block).
Before this test phase, participants performed a short practice run outside the MR scanner. This practice was limited to one block of 16 trials, and the correct answer was provided after every trial. Importantly, the practice run, covered only a small portion of the potential transitions between stimuli during test, hence it was impossible for participants to learn the correct responses from feedback. During MRI scanning, feedback was not provided, and intertrial intervals varied randomly between 6 and 8 s. Participants viewed the test items, back-projected from a LCD projector via a mirror, mounted on the head-coil, angled at $45^{\circ}$. Responses were made using an MR-compatible button-box. Each scanning run comprised one block lasting $\sim 7 \mathrm{~min}$; the experiment consisted of eight runs in total.

\section{$M R$ imaging parameters}

Imaging data were acquired using a 3T MRI scanner (Siemens Trio), with a standard receiver head coil. Twenty-five contiguous axial slices $(3 \mathrm{~mm}$ thickness), tilted to cover target brain areas (eg, RSC, thalamus), were acquired using a $\mathrm{T} 2{ }^{\star}$-weighted gradient echo planar imaging sequence $\left(\mathrm{TR} / \mathrm{TE}=1340 / 30 \mathrm{~ms}\right.$; flip angle $=90^{\circ}$, voxel size $=3 \mathrm{~mm}$ isotropic, FoV $=192 \times 192 \mathrm{~mm}$ ). Before the functional scans, a high-resolution, whole-brain T1-weighted anatomical scan was acquired for each participant $\left(\mathrm{TR} / \mathrm{TE}=15 / 4.2 \mathrm{~ms}\right.$, flip angle $=20^{\circ}$, voxel size $=0.9 \mathrm{~mm}$ isotropic, $\mathrm{FoV}=240 \times 240 \mathrm{~mm}$ ).

\section{fMRI data preprocessing and analysis}

Data analyses were conducted using FSL v5.0 (FMRIB). For each run, the EPI data were skull-stripped (Smith, 2002), realigned to the middle functional volume of the run (Jenkinson et al., 2002), high-pass filtered (100 s) to remove low-frequency scanner artifacts, smoothed with a 5 mm FWHM Gaussian kernel, and transformed to the participant's highresolution anatomical image using boundary-based registration (Greve and Fischl, 2009), before linear registration, with 12 degrees of freedom, to the standard-space template (Montreal Neurological Institute, $n=152)$.

Two separate general linear models (GLMs) were constructed to test: (1) brain regions sensitive to repetition of $\mathrm{HD}$, and (2) the profile of the BOLD response over multiple repetitions of stimuli cueing the same facing direction. First, to examine regions of the brain sensitive to repetition of HD information, three explanatory variables (EVs) were entered into FMRIB's improved linear model of the fMRI time-course data from each of the eight functional runs. These comprised: (1) correct responses to non-repeat HD trials, (2) correct responses to repeat HD trials, and (3) all incorrect responses. This model replicates previous analysis strategies (Baumann and Mattingley, 2010), and benefits from having an equal number of trials in the non-repeat HD and repeat HD regressors (mean number of correct non-repeat HD trials per run $=15.78, \mathrm{SD}=0.42$; mean number of correct repeat-HD trials per run $=15.50, \mathrm{SD}=0.86$ ). To examine the profile of BOLD response over multiple repetitions of $\mathrm{HD}$, a second GLM was implemented in which the repeat HD regressor was separated according to whether a trial comprised a repetition of HD associated with the preceding trial only (one-back repeat HD), or the preceding two trials (two-back repeat HD; mean number of correct oneback repeat $\mathrm{HD}$ trials per run $=9.74, \mathrm{SD}=1.35$; mean number of correct two-back repeat $\mathrm{HD}$ trials per run $=5.76, \mathrm{SD}=1.25$ ). Although the two-back repeat HD EV models those trials in which the preceding two trials comprised the same HD, the participant was required only to make a one-back decision (ie, compare the HD on the current trial with respect to the immediately preceding trial). There was no difference, therefore, in mnemonic demand between the one-back and two-back repeats modeled here. The second model comprised four EVs: (1) correct responses to non-repeat HD trials, (2) correct responses to one-back repeat HD trials, (3) correct responses to two-back repeat HD trials, and (4) all incorrect responses. In both GLMs, the regressors were convolved with a double-gamma HRF and the temporal derivative added. Each participant's eight runs were combined using a fixed-effects model, the resulting images thresholded at $Z>2.3$ to identify contiguous clusters, and a cluster extent of $p<0.05$ applied to control FWE.

Given our a priori hypotheses regarding the role of the RSC and thalamus in HD coding, we examined effects in anatomical regions-of- 
interest (ROIs). The RSC anatomical ROI was created using FreeSurfer 5.0. The participant's $\mathrm{T} 1$ anatomical image was resampled to $1 \mathrm{~mm}$ isotropic resolution, segmented, the gray matter skeleton extracted, and parcellated into separate cortical regions (Destrieux et al., 2010). The RSC was defined as the posterior-ventral part of the cingulate gyrus, providing a mask slightly larger than BA29/30 (ie, RSC proper; Fig. 1e). The RSC masks were then resampled to $0.9 \mathrm{~mm}$ isotropic resolution, and subsequently warped to the standard space template (MNI-152), using the same transformation matrix parameters from the registration of the participant's T1 image to standard space in the functional analyses (Fig. 1f). FMRIB's Integrated Registration and Segmentation Tool (FIRST; Patenaude et al., 2011) was used to segment automatically the thalamus bilaterally in each participant's T1 structural image. The subject-specific thalamic ROIs were then warped to standard space. In our paradigm, different HDs were associated with different landmarks. The recovery of orientation information, therefore, could be interpreted as the recall of landmark representations, and adaptation effects in the RSC and/or thalamus interpreted as reflecting memory for these items. As a control analysis, we tested for adaptation effects also in bilateral hippocampus, a central node for episodic memory (Squire et al., 2004), where one might expect to observe memory-related adaptation effects (Kremers et al., 2014). Subject-specific hippocampal ROIs were identified using FMRIB's FIRST, before being warped to standard space.

For the first GLM, FSL's Featquery was used to extract the average percentage signal change value associated with: (1) "correct responses to non-repeat HD trials $>$ baseline," and (2) "correct responses to repeat HD trials > baseline," in the bilateral RSC and thalamic anatomical ROIs. Given the size of our fMRI sample, it is possible that the data may violate the assumptions of normality, and that the results could be driven by outliers. To control for these possibilities, the percentage signal change data were first rank transformed using the Align Rank Transform (ART) tool (for details, see Wobbrock et al., 2011). Briefly, this method ranks the data according to a specific effect of interest after having "stripped" from it the contributions of other factors in the model (eg, any other main effects, and interactions). Once the data have been aligned and ranked, it can then be submitted to regular parametric ANOVA, with separate models used to examine each individual effect. For example, a two-way ANOVA comprising two main effects and an interaction term would require three separate ANOVA models, two in which the data have been ranked according to each main effect (with the contribution of the other main effect and interaction term removed from these data), and one for the interaction term, where the data have been ranked after the contribution of the main effects have been removed. For our ROI analysis, the resulting rank values were submitted to repeated-measures ANOVAs for the RSC, thalamus, and hippocampus comprising the factors Hemisphere (left; right) and Repetition (non-repeat HD; repeat HD). To examine whether there was further adaptation in the BOLD signal over repeats of the same HD (ie, our second fMRI GLM), the percentage signal change values associated with: (1) "correct responses to one-back repeat HD trials > baseline," and (2) "correct responses to two-back repeat HD trials $>$ baseline" were extracted from the anatomical ROIs, aligned, ranked, and entered into separate ANOVAs, for the RSC, thalamus, and hippocampus comprising the factors Hemisphere (left; right) and Repetition (one-back repeat HD; two-back repeat HD).

Following the targeted anatomical ROI analysis, whole-brain, grouplevel contrasts were implemented to identify other brain regions coding HD. These contrasts were performed using FSL's threshold-free cluster enhancement (TFCE; Smith and Nichols, 2009), with $5 \mathrm{~mm}$ variance smoothing (recommended for small sample sizes), using the Randomize tool (Winkler et al., 2014). The TFCE algorithm computes a voxelwise score that reflects both a voxel's height, and the sum of the spatially contiguous voxels supporting it (Smith and Nichols, 2009, their Eq. 1). The TFCE score, therefore, reflects both the strength of the signal at a given voxel, and its cluster extent. A benefit of this method is that it removes the need for the experimenter to select an arbitrary $Z$ threshold for the group-level cluster statistic. Randomize is a permutation-based method allowing for the analysis of imaging data that, due to small sample size, may not adhere to parametric assumptions (eg, normal distribution). Combined with TFCE, FSL's Randomize constructs a study- specific null distribution of TFCE scores using permutations of the design matrix. For a one-sample $t$ test design matrix (comprising a vector of ones), Randomize flips the signs of this matrix (ie, 1 to -1 ), and permutes all possible combinations of this design (in our case $=2^{9}=512$ permutations). Voxelwise TFCE scores are then computed for each permutation, and the resulting values combined to form a null distribution. This distribution can then be thresholded to control the FWE, for example at the 95th percentile (ie, $p<0.05$ ), and this value then used to threshold the original non-permuted TFCE image. A second benefit of this method, therefore, is that the null distribution is constructed using the observed data. For the first fMRI model, a contrast was implemented to examine brain regions showing repetition suppression associated with repeats, compared with non-repeats, of HD ("non-repeat $\mathrm{HD}>$ repeat HD," ie, where in the brain is there a significant reduction in activity for repeats relative to non-repeats of HD?). The reverse of this contrast was modeled also to examine regions of cortex showing significantly increased BOLD response associated with repeats of HD relative to non-repeats (ie, repetition enhancement; Vass and Epstein, 2013). In the second fMRI GLM, we contrasted one-back repeat HD and two-back repeat HD trials to test whether either of these conditions showed a stronger repetition suppression effect. To localize more precisely effects within the thalamus, we used a stereotactic atlas of this region comprising 53 separate thalamic structures (Morel, 2007), generated in 3D (Krauth et al., 2010), and nonlinearly warped to the MNI-152 template (Jakab et al., 2012).

\section{Results}

\section{Behavioral}

To check whether behavioral performance was equivalent for all orientations and repetition conditions, accuracy and reaction time (RT) data for the one-back task were submitted to an Orientation $\left(0^{\circ}, 90^{\circ}, 180^{\circ}, 270^{\circ}\right) \times$ Repetition (non-repeat HD; repeat HD) repeated-measures ANOVA. Participants performed nearly at ceiling on the task (Fig. 2a), and the ANOVA revealed only a trend toward better performance for the non-repeat HD, relative to the repeat $\mathrm{HD}$ trials $\left(F_{(1,8)}=4.49, p=0.07, \eta_{\mathrm{p}}^{2}=0.36\right)$. There was no effect of orientation, or Orientation $\times$ Repetition interaction $(F$ values $<2.48, p$ values $>0.07)$.

Behavioral priming has been used to examine the underlying mental representations of facing direction (Marchette et al., 2014). Specifically, stimuli cueing repetitions of HD resulted in faster RTs than non-repeats. This was thought to result from the $\mathrm{RT}$ cost of having to switch between different mental representations of facing direction when heading direction was not repeated. Consistent with previous data, we observed a main effect of Repetition $\left(F_{(1,8)}=5.89, p=0.04, \eta_{\mathrm{p}}^{2}=0.42\right)$, reflecting longer RTs for non-repeat HD trials (mean $=1596 \mathrm{~ms}, \mathrm{SEM}=118 \mathrm{~ms}$ ), than for repeat HD trials (mean $=1502 \mathrm{~ms}$, SEM $=121 \mathrm{~ms}$ ). There were no other main effects, or interactions ( $F$ values $<1.54$, $p$ values $>0.22$ ) suggesting that the four different facing directions were equally well learned.

It is possible that the sample size was too small to detect any differences in accuracy and/or RT between the different orientations. To test whether this null effect resulted from a lack of power, we repeated the task (outside of the MRI scanner) in a separate sample of 24 participants ( 14 female; mean age $=24.2$, range: $20-31$ ). The behavioral procedure for the additional participants was the same as reported for the fMRI task with the exception that the intertrial interval was adjusted to $2 \mathrm{~s}$; target image presentation time was maintained at $5 \mathrm{~s}$.

Mean accuracy and RTs for the one-back task were submitted to Orientation $\left(0^{\circ}, 90^{\circ}, 180^{\circ}, 270^{\circ}\right) \times$ Repetition (non-repeat $\mathrm{HD}$; repeat $\mathrm{HD})$ repeated-measures ANOVAs. For accuracy scores, we observed a main effect of Repetition, $\left(F_{(1,23)}=8.23\right.$, $\left.p=0.01, \eta_{\mathrm{p}}^{2}=0.26\right)$, modulated by a significant Orientation $\times$ 

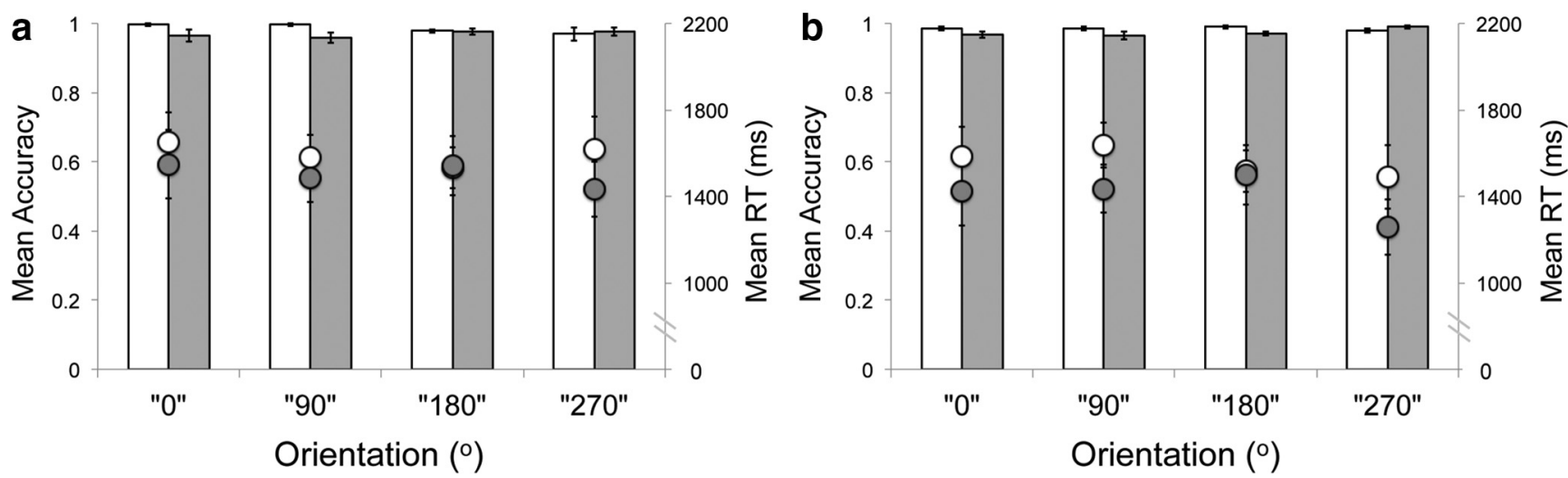

\section{$\square$ Non-repeat HD Accuracy $\square$ Repeat HD Accuracy $\bigcirc$ Non-repeat HD RT $\bigcirc$ Repeat HD RT}

Figure 2. Behavioral performance (mean accuracy and RT \pm SEM) on the one-back task, separated according to orientation and repetition. $\boldsymbol{a}$, The fMRI sample $(n=9)$ showed very accurate performance on this task, with mean accuracy and RT matched across different orientations; $(\boldsymbol{b})$ the excellent behavioral performance was replicated in a larger sample $(n=24)$, where these participants showed a small benefit in RT for stimuli cueing the $270^{\circ}$ orientation.

\section{Retrosplenial cortex}
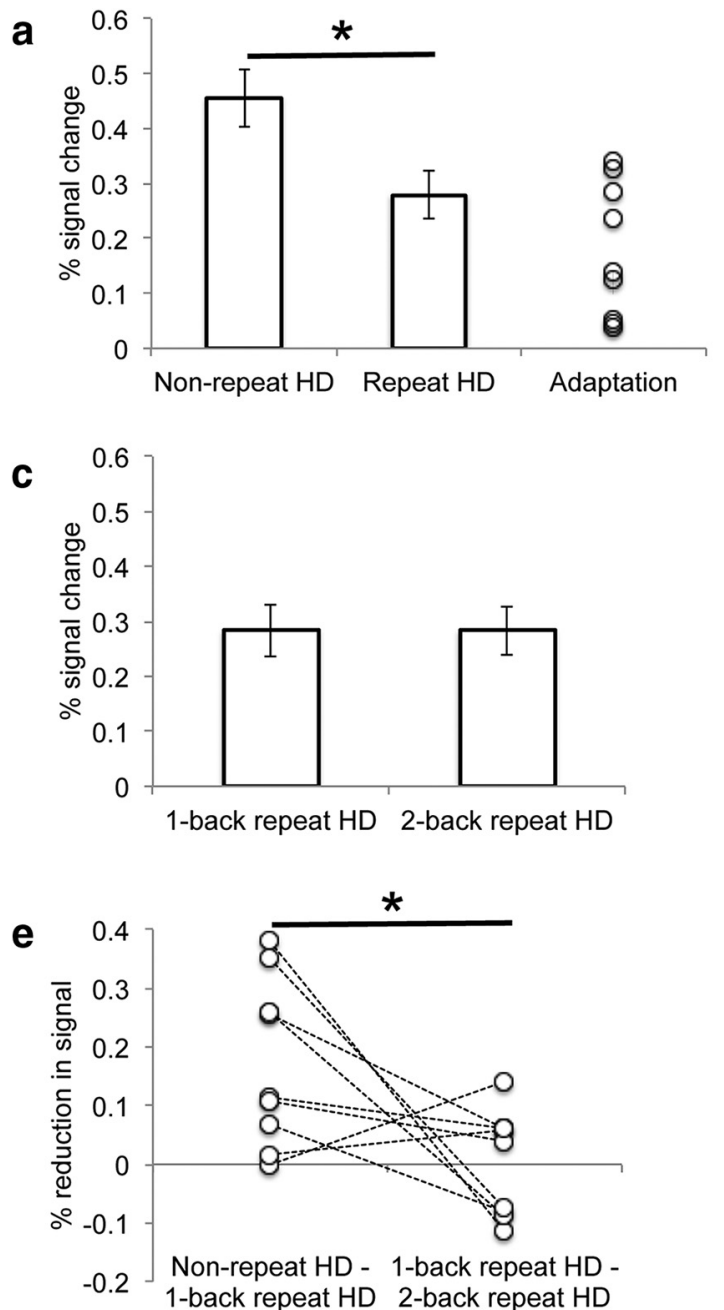

Thalamus
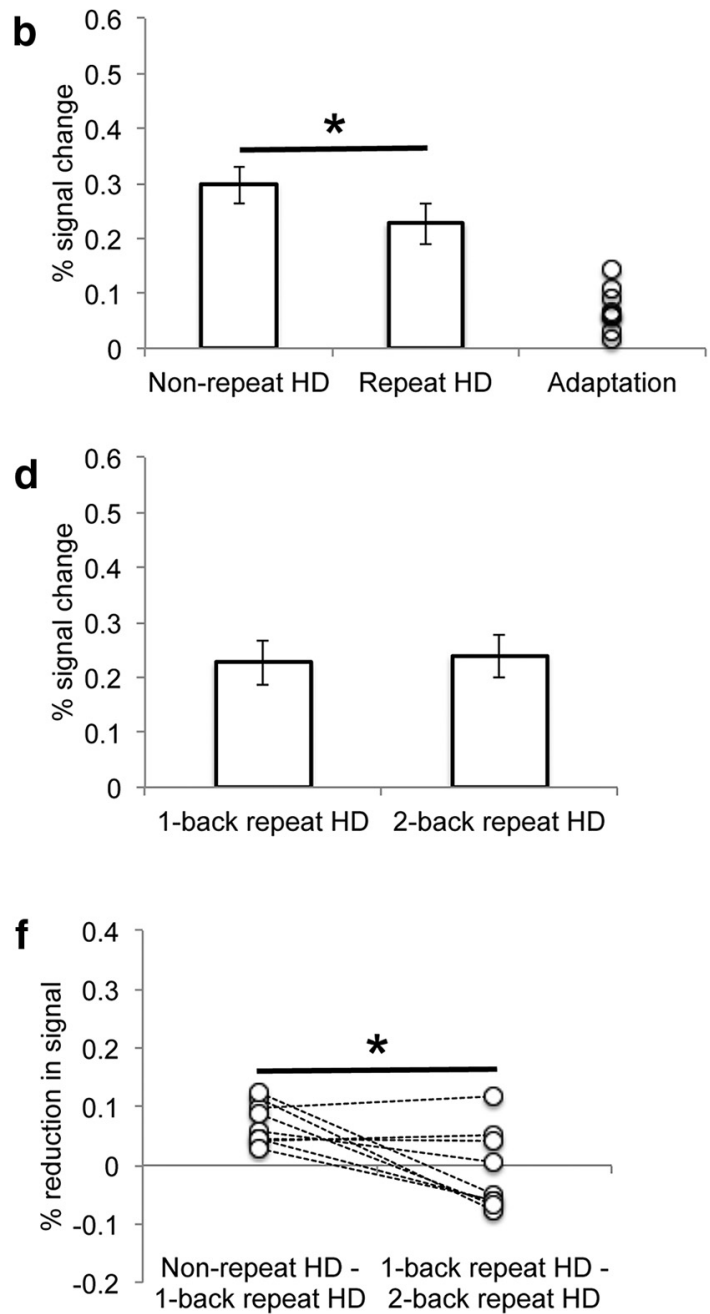

Figure 3. Group average percentage signal change values (mean \pm SEM) extracted from anatomical ROIs. $\boldsymbol{a}$, In bilateral RSC, significant repetition suppression was associated with repeat $H D$ relative to non-repeat HD trials. The percentage signal adaptation between these two conditions for each participant is also provided; positive values demonstrate that repetition of $H D$ led to a reduction of the BOLD signal; (b) the same adaptation effect was evident also in bilateral thalamus; (c) in RSC attenuation did not continue over multiple repetitions of stimuli cueing the same HD; (d) and this plateau was apparent also in the thalamus. To test more directly for evidence of a plateau in the BOLD response after the first repetition of HD, the difference in activity between non-repeat $H D$ and one-back repeat $H D$ was compared with the difference in activity between one-back repeat HD and two-back repeat HD conditions. In both $(\boldsymbol{e}) \mathrm{RSC}$, and $(\boldsymbol{f})$ the thalamus, significantly greater adaptation was found after the first repeat of HD compared with when HD was repeated over multiple trials. Individual-subject data are displayed here, with dashed lines connecting each subject's data points $\left({ }^{*} p<0.05\right)$. 

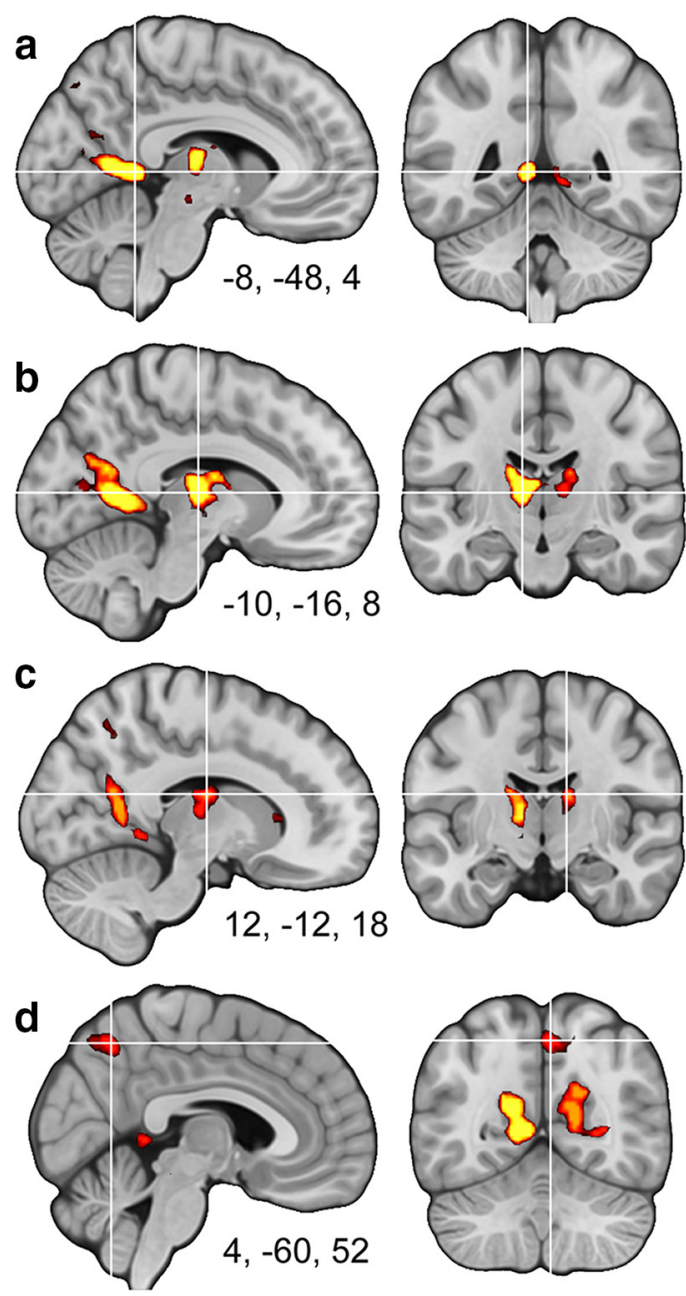

FWE1-P
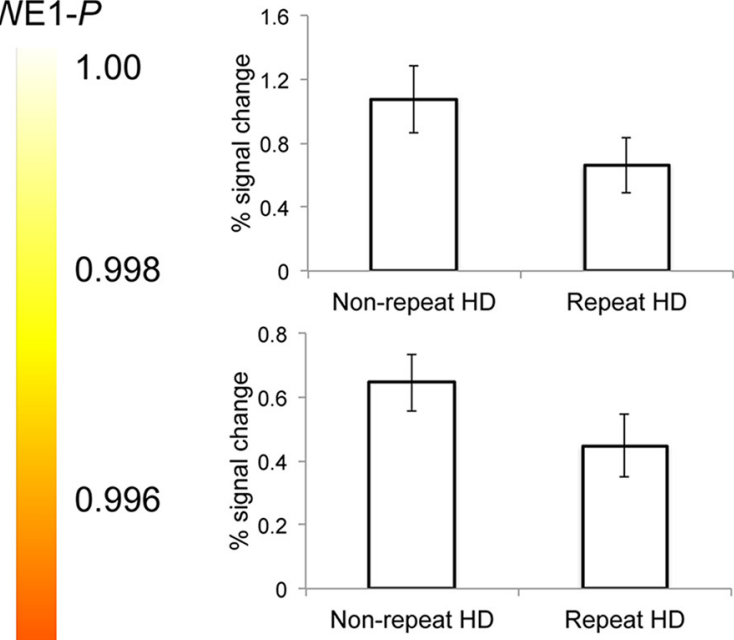

0.994

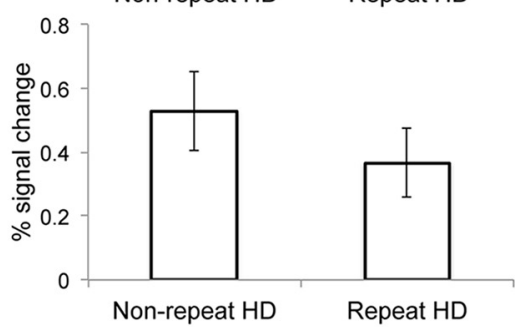

0.992

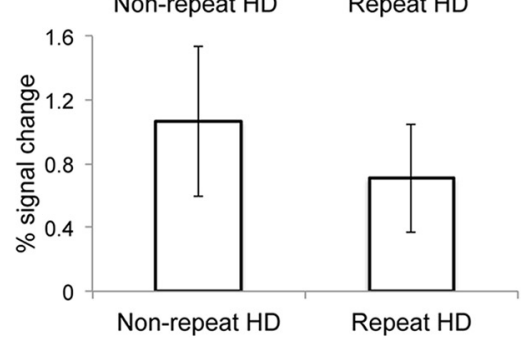

Figure 4. Whole-brain contrast image (non-repeat HD $>$ repeat HD) with corresponding plots of mean percentage signal change values \pm SEM extracted from the peak voxel in each cluster. Supporting the ROI analyses, significant repetition suppression was evident in the whole-brain contrast image in a number of regions including: (a) left RSC, (b) left thalamus, (c) right thalamus, and (d) precuneus. TFCE results are displayed on the MNI template brain, using a threshold of $p<0.01$, FWE-corrected.

Repetition interaction $\left(F_{(3,69)}=3.18, p=0.03, \eta_{\mathrm{p}}^{2}=0.12\right)$; there was no main effect, however, of Orientation $\left(F_{(3,69)}=1.28, p=\right.$ $\left.0.29, \eta_{\mathrm{p}}^{2}=0.05\right)$. Simple main effects revealed no differences in accuracy across the different orientations for non-repeat, and repeat HDs $(F$ values $<2.35$, $p$ values $>0.09)$. The Orientation $\times$ Repetition interaction resulted from significantly greater accuracy for non-repeat HD relative to repeat HD trials in the $180^{\circ}$ orientation $\left(F_{(1,23)}=10.13, p=0.004\right)$. A similar pattern of data were evident for $0^{\circ}$ and $90^{\circ}$ orientations, however, these comparisons did not reach statistical significance $\left(F_{(1,23)}=4.20, p=0.05 ; F_{(1,23)}=4.02, p=0.06\right.$, respectively); there was no difference in the level of accuracy between non-repeat and repeat $\mathrm{HD}$ trials for the $270^{\circ}$ orientation $\left(F_{(1,23)}=1.81, p=0.19\right.$; Fig. $\left.2 b\right)$.

As in the fMRI study, RTs were faster for repeat versus nonrepeat trials (main effect of Repetition: $F_{(1,23)}=17.39, p<0.001$, $\left.\eta_{\mathrm{p}}^{2}=0.43\right)$. In addition, we observed a main effect of Orientation $\left(F_{(3,69)}=12.90, p<0.001, \eta_{\mathrm{p}}^{2}=0.36\right)$, qualified by an Orientation $\times$ Repetition $\left(F_{(3,69)}=6.79, p<0.001, \eta_{\mathrm{p}}^{2}=0.23\right)$ interaction. To further explore these effects, we tested whether RT differed across different orientations for non-repeat, and repeat HD conditions. For the non-repeat HD condition, responses for stimuli cueing $180^{\circ}$ and $270^{\circ}$ orientations were quicker than for stimuli cueing $0^{\circ}$ and $90^{\circ}$ (all $t$ values $>2.93, p$ values $<0.008$ ).
For the repeat HD condition, RTs were faster for stimuli cueing the $270^{\circ}$ orientation relative to all other orientations (all $t$ values $>4.39$, $p$ values $<0.001$ ). Furthermore, simple main effects comparing RT between non-repeat and repeat HD conditions for each individual orientation revealed significantly quicker responses for repeat $\mathrm{HD}$ trials in $0^{\circ}, 90^{\circ}$, and $270^{\circ}$ orientations (all $F$ values $>8.21$, $p$ values $<0.01)$. Although there was no statistically significant difference in RTs between non-repeat, and repeat $\mathrm{HD}$ conditions for the $180^{\circ}$ orientation $\left(F_{(1,23)}=0.18, p=\right.$ $0.67)$, the pattern of data were in the same direction with faster responses for repeat $\mathrm{HD}$ trials $($ mean $=1498 \mathrm{~ms}, \mathrm{SEM}=61 \mathrm{~ms}$ ) compared with non-repeat HD trials $($ mean $=1516 \mathrm{~ms}, \mathrm{SEM}=$ $62 \mathrm{~ms})$.

In summary, in a separate behavioral experiment we found that, in terms of accuracy, performance was again nearly at ceiling and was matched across the four different orientations in the VE. For RT, there was a small benefit in RT for stimuli cueing the $270^{\circ}$ orientation. This effect, however, was not consistent across both repetition conditions, and $\mathrm{RT}$ s were faster for repeat trials in all four orientations. Together, these results confirm the effects of the fMRI experiment and demonstrate the behavioral advantage of orientation priming, which was not driven by a particular orientation. 


\section{fMRI: ROI analysis}

In the following section, we detail the analysis of: (1) percentage signal change values associated with non-repeat, and repeat HD trials; (2) percentage signal change values associated with oneback repeat HD and two-back repeat HD; and (3) the difference in percentage signal change values between non-repeat HD and one-back repeat HD trials versus the difference between oneback repeat HD and two-back repeat HD trials. For all analyses, we used participant-specific anatomical ROIs comprising the left and right RSC, left and right thalamus, and left and right hippocampus; results are described in this order.

In RSC there was a significant main effect of Repetition, with non-repeat $\mathrm{HD}$ trials associated with greater percentage signal change than repeat $\mathrm{HD}$ trials $\left(F_{(1,8)}=22.73, p=0.001, \eta_{\mathrm{p}}^{2}=0.74\right.$; Fig. $3 a)$. Repetition condition interacted with Hemisphere $\left(F_{(1,8)}\right.$ $\left.=10.26, p=0.013, \eta_{\mathrm{p}}^{2}=0.56\right)$, and follow-up nonparametric Wilcoxon signed-rank tests showed a trend toward greater activity associated with repeat-HD trials in right, relative to left RSC $[Z=1.84, p=0.066$ (two-tailed), $r=0.61]$. In the thalamic ROIs, we observed a similar pattern of data (Fig. $3 b$ ), with significantly greater activity for non-repeat HD trials relative to repeat HD trials $\left(F_{(1,8)}=20.16, p=0.002, \eta_{p}^{2}=0.72\right)$. There was no effect of Hemisphere, and this factor did not interact with Repetition ( $F$ values $<2.80$, $p$ values $>0.12$ ). In contrast, in the hippocampus there were no significant effects ( $F$ values $<1.14$, $p$ values $>0.30$ ).

Rodent data have shown that the firing rate of HD cells reaches a stable plateau after $\sim 4 \mathrm{~s}$ when the animal is held stationary (Shinder and Taube, 2014). To determine whether similar dynamics might be present in humans, we asked whether the profile of the BOLD response changes over multiple repetitions of the same HD. For both the RSC (Fig. 3c) and thalamus (Fig. $3 d$ ), there was no evidence that percentage signal change values differed according to Hemisphere (left; right), Repetition (oneback repeat HD; two-back repeat HD), or a Hemisphere $\times$ Repetition interaction ( $F$ values $<1.14$, $p$ values $>0.31$ ). Likewise, percentage signal change values in the hippocampus did not differ according to these factors ( $F$ values $<2.63$, $p$ values $>0.13$ )

To provide stronger support for the notion that the BOLD signal plateaus over repetitions of stimuli cueing the same HD, we compared the difference in activity between the non-repeat HD and oneback repeat HD trials with the difference between the one-back repeat $\mathrm{HD}$ and two-back repeat $\mathrm{HD}$ trials. In both the RSC (Fig. 3e) and thalamus (Fig. 3f), nonparametric Wilcoxon signed-rank tests revealed significantly greater adaptation when HD was repeated for the first time ("non-repeat HD - one-back repeat HD"), compared with subsequent repetitions of HD ["one-back repeat HD - twoback repeat HD"'; RSC: $Z=2.07, p=0.02$ (one-tailed), $r=0.69$; thalamus: $Z=2.07, p=0.02$ (one-tailed), $r=0.69$ ]. Consistent with the results of the non-repeat HD versus repeat-HD comparison reported above, in the hippocampus there was no difference in the degree of adaptation between "non-repeat HD - one-back repeat HD" and "one-back repeat HD - two-back repeat HD" $[Z=-0.53, p=0.30$ (one-tailed), $r=0.18]$.

To summarize, in anatomical ROIs comprising the RSC and thalamus, significant repetition suppression was observed following repeats of $\mathrm{HD}$, relative to when $\mathrm{HD}$ direction was changed. Moreover, during a second repetition of the same HD, the BOLD signal remained suppressed but did not show any evidence of further attenuation. There was no evidence of adaptation, however, in the hippocampus associated with repeated HD.
Table 1. MNI coordinates of peak voxels and local maxima in the whole-brain repetition suppression analysis (non-repeat HD $>$ repeat HD; $p<0.01$, FWEcorrected)

\begin{tabular}{|c|c|c|c|c|c|c|}
\hline \multirow[b]{2}{*}{ Region } & \multirow[b]{2}{*}{ Voxels } & \multirow[b]{2}{*}{$1-p$ value } & \multirow[b]{2}{*}{$t$} & \multicolumn{3}{|c|}{ MNI coordinates } \\
\hline & & & & $x$ & $y$ & $z$ \\
\hline \multirow[t]{12}{*}{ Left retrosplenial cortex } & 1444 & 0.998 & 7.37 & -8 & -48 & 4 \\
\hline & & & 5.49 & 16 & -56 & 10 \\
\hline & & & 4.84 & -12 & -60 & 2 \\
\hline & & & 4.60 & -14 & -70 & 22 \\
\hline & & & 4.59 & -8 & -58 & 2 \\
\hline & & & 4.58 & -6 & -62 & 4 \\
\hline & & & 4.52 & -8 & -54 & 0 \\
\hline & & 0.996 & 5.38 & 10 & -46 & -2 \\
\hline & & & 5.17 & 28 & -56 & 4 \\
\hline & & & 4.61 & 16 & -56 & 6 \\
\hline & & & 4.52 & 12 & -54 & 4 \\
\hline & & & 4.40 & 16 & -62 & 8 \\
\hline \multirow[t]{8}{*}{ Left thalamus } & 871 & 0.998 & 7.13 & -10 & -16 & 8 \\
\hline & & & 5.18 & -10 & -8 & 16 \\
\hline & & & 5.09 & -18 & 10 & -8 \\
\hline & & & 4.94 & -8 & -18 & 4 \\
\hline & & & 4.90 & -2 & -20 & 10 \\
\hline & & 0.996 & 4.30 & 0 & -24 & 10 \\
\hline & & & 4.22 & -12 & -2 & 12 \\
\hline & & 0.990 & 3.68 & -10 & -14 & -4 \\
\hline \multirow[t]{3}{*}{ Right thalamus } & 158 & 0.996 & 5.66 & 12 & -12 & 18 \\
\hline & & & 5.33 & 14 & -14 & 16 \\
\hline & & 0.994 & 5.35 & 12 & -16 & 8 \\
\hline \multirow[t]{9}{*}{ Right precuneus } & 301 & 0.994 & 5.42 & 4 & -60 & 52 \\
\hline & & & 4.84 & 4 & -60 & 48 \\
\hline & & & 4.70 & 6 & -64 & 50 \\
\hline & & 0.992 & 4.34 & -2 & -78 & 46 \\
\hline & & & 4.32 & -6 & -80 & 44 \\
\hline & & & 4.32 & 16 & -62 & 52 \\
\hline & & & 4.26 & 2 & -68 & 46 \\
\hline & & & 4.24 & 10 & -58 & 46 \\
\hline & & 0.990 & 3.96 & -2 & -80 & 42 \\
\hline \multirow[t]{6}{*}{ Right caudate } & 228 & 0.996 & 5.70 & 18 & 18 & 6 \\
\hline & & & 5.66 & 18 & 16 & 4 \\
\hline & & 0.994 & 4.87 & 18 & 16 & -4 \\
\hline & & & 4.82 & 20 & 12 & -2 \\
\hline & & 0.992 & 5.24 & 22 & 4 & -10 \\
\hline & & 0.990 & 4.29 & 18 & 26 & -4 \\
\hline \multirow[t]{4}{*}{ Brain stem } & 87 & 0.992 & 5.35 & -4 & -22 & -10 \\
\hline & & & 4.55 & 0 & -26 & -14 \\
\hline & & 0.990 & 3.92 & -2 & -24 & -18 \\
\hline & & & 3.76 & 2 & -32 & -16 \\
\hline $\begin{array}{l}\text { Superior temporal gyrus } \\
\text { (posterior division) }\end{array}$ & 42 & 0.994 & 6.56 & 46 & -36 & 4 \\
\hline \multirow[t]{3}{*}{ Inferior frontal gyrus } & 15 & 0.990 & 6.64 & -54 & 10 & -2 \\
\hline & & & 6.57 & -56 & 8 & -2 \\
\hline & & & 6.25 & -58 & 4 & 0 \\
\hline
\end{tabular}

\section{fMRI: whole-brain analysis}

To determine whether regions of cortex outside our predefined ROIs were sensitive to repetition of HD, we performed a wholebrain analysis. Using a voxelwise threshold of $p<0.05$ (FWEcorrected), for the non-repeat $\mathrm{HD}>$ repeat $\mathrm{HD}$ contrast we found a large cluster of activity, centered on the left RSC, which encompassed a number of brain regions, including the right RSC, bilateral thalamus, precuneus, and putamen, and a smaller cluster in the temporal pole. Given the large spatial extent of the first cluster, the image was thresholded at $p<0.01$ (FWE-corrected) to see in which regions the effect of this contrast was strongest. Supporting the ROI analysis, we observed separate clusters in left RSC (which included a local maximum in the right RSC) and left and right thalamus. Moreover, there was activity also in the precuneus (Fig. 4; Table 1). The reverse of this contrast ("repeat 
HD > non-repeat HD"; ie, repetition enhancement) was not associated with any regions of significantly increased BOLD response, at either threshold.

Using the Morel (2007) thalamic atlas, we identified the peak coordinate of the left thalamus cluster in the ventral division of the ventral lateral posterior nucleus. As can be seen in Figure 5, this cluster spanned a number of other thalamic subregions including the dorsal division of the ventral lateral posterior nucleus, the central lateral nucleus, the lateral dorsal and lateral posterior nuclei, the parvocellular division of the mediodorsal nucleus, and the posterior division of the ventral posterior lateral nucleus. The cluster in the right thalamus spanned the same thalamic subregions as the left. Its peak, however, was located at the border of the dorsal division of the ventral lateral posterior nucleus.

To examine the consistency of our effects, we investigated the repetition suppression contrast at the single-subject level. Seven of the nine participants showed significant clusters in the RSC (Fig. 6a). Eight of the nine subjects showed repetition suppression in the thalamus (masked with their own bilateral thalamic ROI) associated with repeating HD (Fig. 6b), with this contrast surviving correction for multiple comparisons in six subjects. The observed wholebrain effects in the RSC and thalamus, therefore, reflect consistent patterns of activity across nearly the entire sample.

Finally, consistent with the targeted ROI analyses, there were no differences in the level of activity associated with oneback repeat HD and two-back repeat HD trials anywhere in the brain.

\section{Discussion}

We used repetition suppression to examine brain regions coding HD in a VE using global reference frame cues. Unlike previous studies, orientation was learned using both visual and body-based cues; during MRI scanning only visual cues were provided for orientation. In bilateral RSC and thalamic ROIs, the BOLD signal attenuated in association with repeating HD. Consistent with rodent electrophysiology, this signal plateaued over multiple repetitions of the same facing direction. These data were supported by whole-brain analyses showing adaptation also in the precuneus. Here, we show evidence that: (1) the thalamus codes facing direction, (2) HD coding occurs in anatomically defined RSC (rather than retrosplenial complex), and (3) participants integrate HD across different locales using global reference frame cues.

In rats, both body-based and visual cues contribute to accurate orientation coding. The $\mathrm{ADN}$ of the thalamus is thought to integrate this information (Taube, 1995), as it receives projec-

\section{Left thalamus cluster}
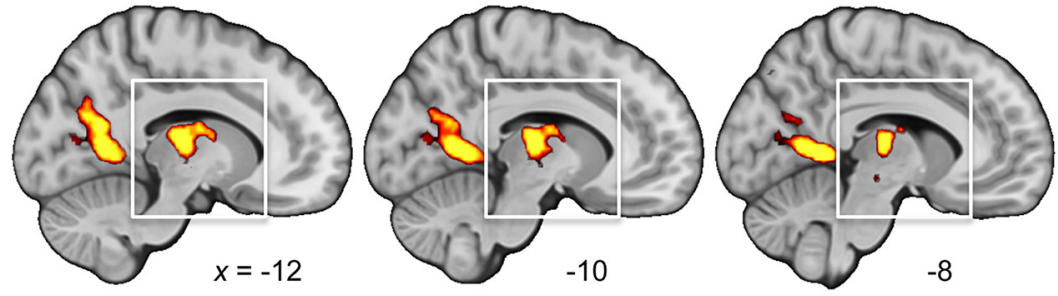

Morel thalamic atlas overlay
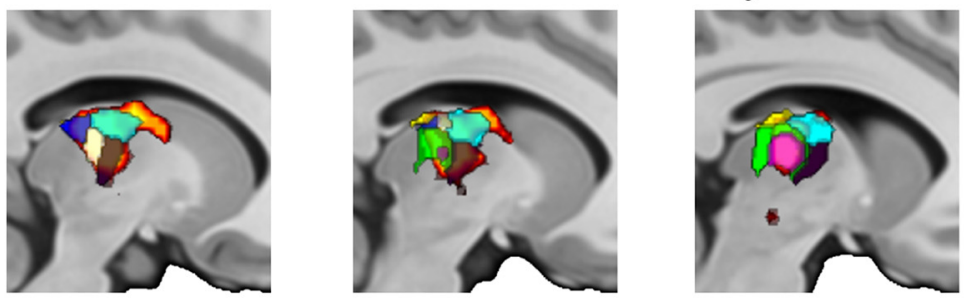

Right thalamus cluster
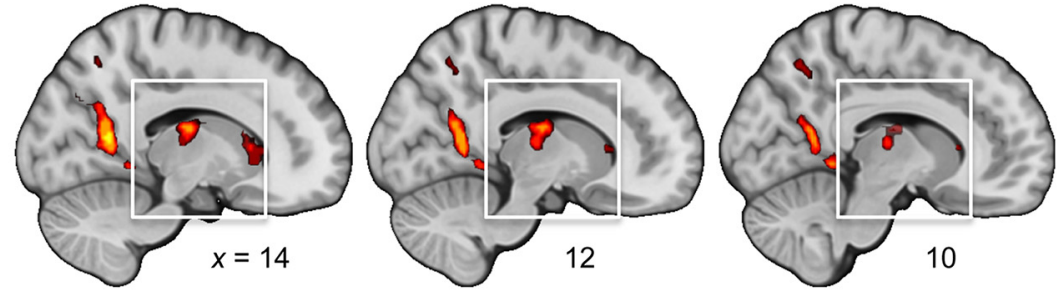

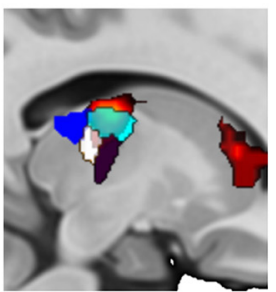

$\mathrm{CL}$

Figure 5. Significant repetition suppression effect in bilateral thalamus overlaid with the Morel (2007) thalamic atlas. According to this atlas, the peak coordinate of left thalamus cluster $(-10,-16,8$; displayed center in the top row of the figure, accompanied by adjacent sagittal slices) was located in the ventral division of the ventral lateral posterior nucleus [VLpv; the second row of the figure comprises enlarged views of the left thalamus with color-coded overlay of the Morel (2007) atlas]. This cluster extended to the dorsal division of the ventral lateral posterior nucleus (VLpd), the central lateral nucleus (CL), the lateral dorsal nucleus (LD), lateral posterior nucleus (LP), the parvocellular division of the mediodorsal nucleus (MDpc), and the posterior division of the ventral posterior lateral nucleus (VPLp). The right thalamus cluster $(12,-12,18$; third row center, again displayed center alongside two adjacent sagittal slices) spanned the same thalamic subregions. The peak, however, was located on the border of the dorsal division of the ventral lateral posterior nucleus (4th row of figure). Results are displayed on the MNI template brain, with TFCE statistical map thresholded, $p<0.01$ FWE-corrected. tions from vestibular regions and shares reciprocal connections with visual processing areas (RSC/PoS; Clark et al., 2010; Yoder and Taube, 2014). Consistent with these data, previous human fMRI HD studies using VEs, in which only visual cues were provided, observed $\mathrm{HD}$ computations in visual processing regions (RSC/subiculum; Baumann and Mattingley, 2010; Chadwick et al., 2015; Marchette et al., 2014). Here, we provided also bodybased cues, and were therefore more likely to tax brain regions integrating this information (ie, the thalamus). Given that only visual cues for $\mathrm{HD}$ were presented during test, we propose that these led to a recapitulation of the body-based information en- 


\section{a Repetition suppression in the RSC in individual participants}

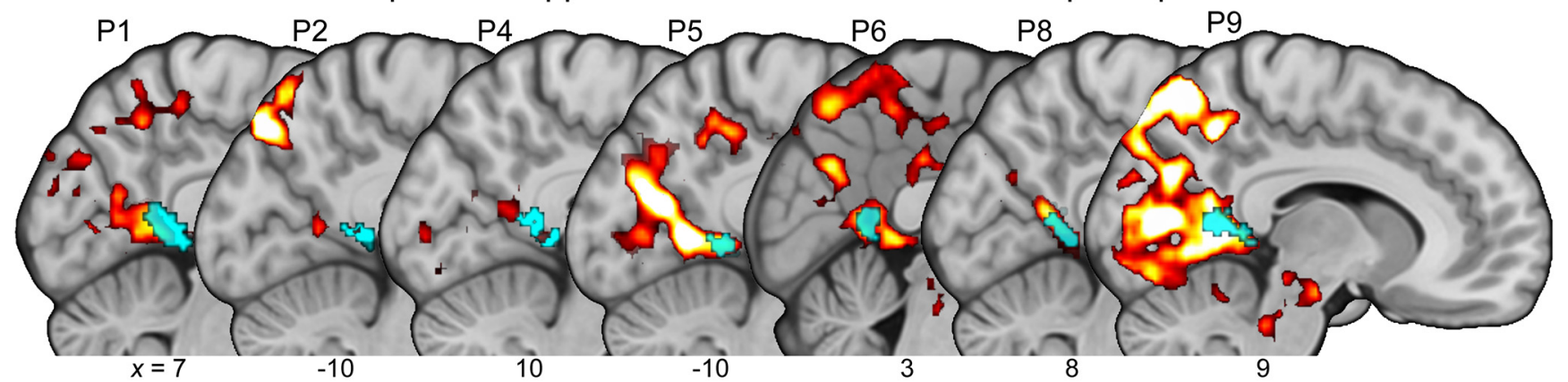

b

Repetition suppression in the thalamus in individual participants
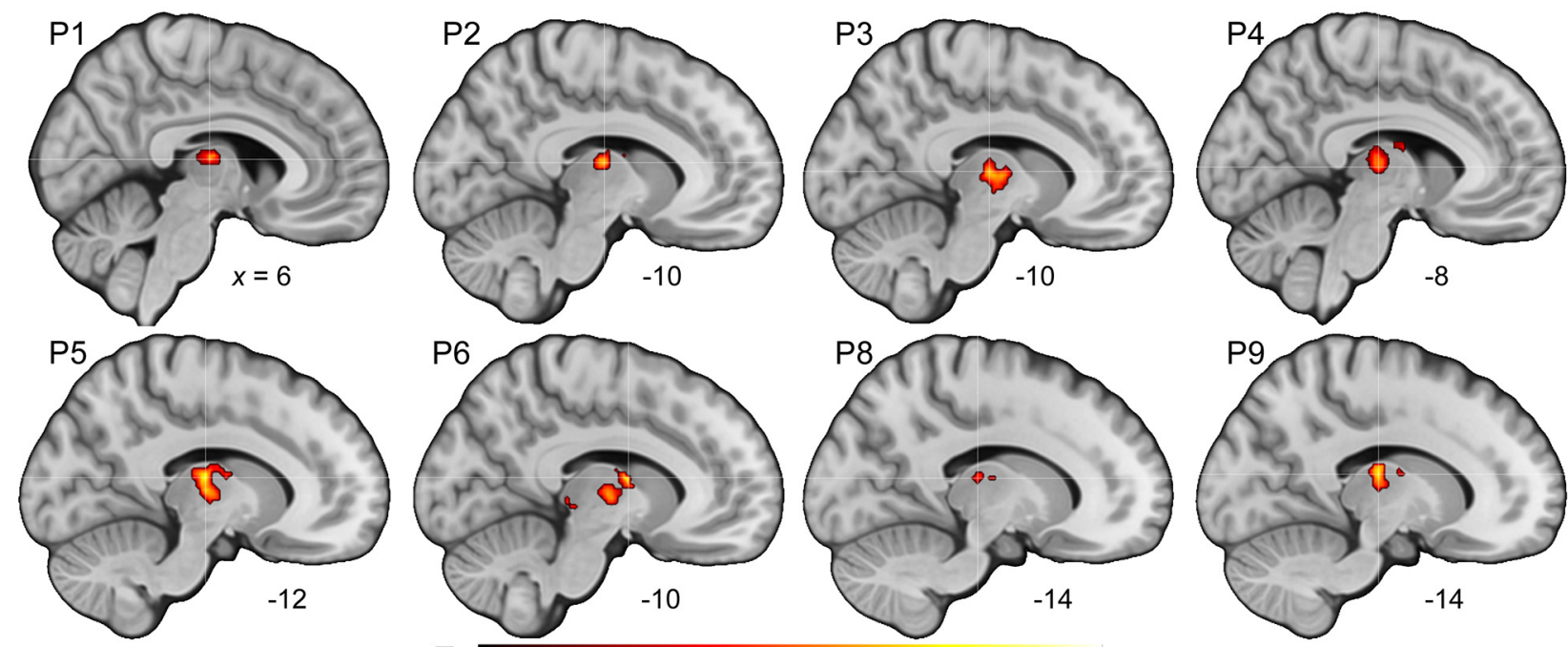

Z

1
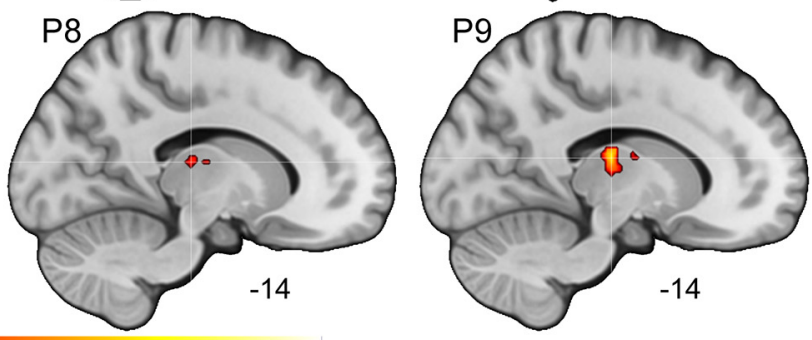

Figure 6. Consistency of repetition suppression in the RSC and thalamus in individual participants. $\boldsymbol{a}$, Significant clusters of activity in the RSC were evident in seven of the nine participants. Clusters are significant at the whole-brain level $(Z>2.3, p<0.05$ ), overlaid (cyan) with the individual participant's RSC anatomical ROI. $\boldsymbol{b}$, Peak voxel in the thalamus associated with the repetition suppression contrast at the single-subject level. Eight participants showed repetition suppression when HD was repeated over consecutive trials. This effect was significant in six participants using a small volume correction comprising the participant-specific bilateral thalamus mask ( $Z>2.3, p<0.05$, corrected for multiple comparisons); this effect did not survive correction for multiple comparisons in participants (P)2 and P8 (images for these participants displayed at $p=0.01$, uncorrected).

coded during learning of the VE (Tulving and Thomson, 1973; Johnson and Rugg, 2007; Wimber et al., 2012), specifically the participant's physical orientation relative to the global landmarks. Because both cue types were available during learning, however, it is not possible to assess the relative contribution of RSC and thalamus to the processing of visual and body-based cues, respectively. In future neuroimaging studies it will be important to separate these different sources of HD information, and to make direct comparisons between "visual" and "visual plus vestibular" cues to help elucidate the precise roles of these brain regions in $\mathrm{HD}$ coding.

Our VE was designed to provide strong body-based cues for $\mathrm{HD}$, with cardinal directions denoted by unique visual landmarks arranged in orthogonal bodily orientations. Moreover, participants monitored their orientation during navigation. These factors may have led to stronger body-based cues than those generated during navigation in the real world. A recent fMRI study, using stimuli comprising images of a real-world environment familiar to the participants (ie, encoded with body-based cues), found that the presubiculum, but not the thalamus, coded facing direction (Vass and Epstein, 2013). Navigation of this larger environment likely required participants to make a greater number of turns, leading to noisier body-based cues (Souman et al., 2009). Furthermore, computational models propose that under normal circumstances (ie, when not conflicting greatly with body-based cues) visual, rather than body-based cues, dominate the HD signal (Knight et al., 2014). Together, these factors may reduce the strength of the body-based cues during navigation in real-world environments, and potentially explain the discrepancy with the findings reported here. In summary, although we observed thalamic HD computations, the scale and layout of the environment, as well as attentional demands, may influence the contribution of this region to HD coding.

Using the Morel (2007) atlas, group-level bilateral thalamic cluster peaks were identified in the ventral/dorsal divisions of the ventral lateral posterior nucleus, rather than the ADN. In macaques, tracer studies show that the ventral lateral region of the thalamus projects to area V6a, a region involved in organizing visual and motor responses (Gamberini et al., 2015). Moreover, human area $\mathrm{V} 6+$ shares strong interconnections with $\mathrm{V} 6 \mathrm{a}$, which is sensitive to changes in head orientation (Arnoldussen et al., 2011). Accordingly, V6a may aid integration of visual and vestibular information to distinguish changes in head orientation relative to the environment, helping to encode facing direction. For 
several reasons, however, we express caution at attributing our group-level effect to specific thalamic subregions. First, the cluster peaks varied across subjects. Second, adaptation was observed in ROIs comprising the entire thalamus, meaning subthreshold effects were likely present in other regions. Finally, given the spatial resolution of our imaging data, we likely sampled over a number of subregions, and were not sensitive to smaller structures [eg, the volume of bilateral ADN in the $1 \mathrm{~mm}$ Morel (2007) atlas is only $16 \mathrm{~mm}^{3}$; Krauth et al., 2010; Jakab et al., 2012].

A second key finding was the demonstration of HD coding using global reference frame cues. Although Marchette et al. (2014) found that RSC was insensitive to this information, their participants were not instructed to attend to the global landmarks, and knowledge of landmark direction was not assessed. The different task demands, therefore, may explain the discrepancy in reference frame coding. Behavioral experiments suggest separate local spatial representations can be integrated into configural representations (Montello and Pick, 1993). For example, after walking around a complex VE, participants pointed more accurately to distant targets when aligned either with local or global reference frames (Meilinger et al., 2014). Similarly, separate spatial representations for individual environments are formed even when learned together (Han and Becker, 2014). Both local, and global reference frames, therefore, may operate in parallel, and it remains to be tested as to whether HD coding changes on a per-trial basis as a function of task instructions (ie, attend either to local or global reference frame cues).

An influential conceptualization of RSC function posits that this region translates between egocentric and allocentric spatial representations, contained in posterior parietal cortex and the medial temporal lobe, respectively (Byrne et al., 2007). Supporting this idea, imaging studies have shown RSC activity is modulated when translating between reference frames (Zhang et al., 2012; Dhindsa et al., 2014). It remains unclear, however, how these representations are synchronized. Alexander and Nitz (2015) found different cells in the rat RSC code for egocentric or allocentric reference frames, and conjunctions of these representations. Consequently, our findings of global reference frame coding, and those reporting HD coding in separate local reference frames (Marchette et al., 2014), are not contradictory; they may simply reflect different forms of spatial mapping supported by different cell populations in the RSC. In terms of how these representations are coordinated, a recent fMRI study found a positive correlation between RSC activity and the degree of landmark permanence (Auger et al., 2012), and these fixed landmark positions may help align different reference frames. This function is consistent with the deficits reported after RSC lesions (Maguire, 2001). Specifically, although patients recognize familiar landmarks, they cannot derive from them topographical information that supports navigation (Ino et al., 2002). We speculate, therefore, that the RSC adaptation observed here is driven by representations of allocentric HD, rather than memory for the landmark itself. Moreover, although we cannot rule out a memory processing explanation for the effects reported here, unlike previous studies (Kremers et al., 2014), we found no evidence of adaptation in the hippocampus, a key node for episodic memory (Squire et al., 2004).

The notion that subdivisions of parietal cortex contain cells coding in egocentric and allocentric reference frames is supported by evidence of adaptation in the precuneus. Although classically implicated in egocentric coding (Wolbers et al., 2008), our data implicate this region in allocentric HD coding. Although changes in allocentric HD may also result in an associated update in egocentric coordinates, our findings are in agreement with rodent data showing that posterior parietal cortex cells code allocentric HD (Wilber et al., 2014). Moreover, the primate posterior parietal cortex provides both egocentric and world-centered codes for external locations (Snyder et al., 1998). Consequently, it is also unclear at present whether the transformation between egocentric and allocentric coordinates is exclusive to RSC.

Finally, it has been suggested that fMRI may not be appropriate to investigate spatial navigation because participants lie motionless in the scanner (Taube et al., 2013). Furthermore, in rats HD cell firing does not adapt when the same orientation is adopted repeatedly, meaning that fMRI repetition suppression is potentially insensitive to this effect. However, when rats adopt a new head orientation after passive rotation, and their heads are kept stationary, HD firing rates are maximal for the first $100 \mathrm{~ms}$ before adapting over the next $4 \mathrm{~s}$ to stabilize at $42 \%$ of their initial values (Shinder and Taube, 2014). Consistent with these data, and supporting previous fMRI results (Baumann and Mattingley, 2010), we observed adaptation in key structures of the rodent HD network associated with repetition of HD information. Furthermore, the BOLD response plateaued, reducing when $\mathrm{HD}$ was first repeated but not over multiple repetitions. Even though there is no simple relationship between neural firing rates and the hemodynamic response (Ekstrom et al., 2009), and a shorter duration, rather than reduced frequency, of neural firing could explain the observed fMRI adaptation (Grill-Spector et al., 2006), our data accord with rodent electrophysiology. FMRI, in combination with realistic training procedures, therefore, appears to be a viable method to understand neural mechanisms supporting spatial navigation.

\section{References}

Alexander AS, Nitz DA (2015) Retrosplenial cortex maps the conjunction of internal and external spaces. Nat Neurosci 18:1143-1151. CrossRef Medline

Arnoldussen DM, Goossens J, van den Berg AV (2011) Adjacent visual representations of self-motion in different reference frames. Proc Natl Acad Sci U S A 108:11668-11673. CrossRef Medline

Auger SD, Mullally SL, Maguire EA (2012) Retrosplenial cortex codes for permanent landmarks. PloS One 7:e43620. CrossRef Medline

Baumann O, Mattingley JB (2010) Medial parietal cortex encodes perceived heading direction in humans. J Neurosci 30:12897-12901. CrossRef Medline

Byrne P, Becker S, Burgess N (2007) Remembering the past and imagining the future: a neural model of spatial memory and imagery. Psychol Rev 114:340-375. CrossRef Medline

Chadwick MJ, Jolly AE, Amos DP, Hassabis D, Spiers HJ (2015) A goal direction signal in the human entorhinal/subicular region. Curr Biol 25: 87-92. CrossRef Medline

Clark BJ, Bassett JP, Wang SS, Taube JS (2010) Impaired head direction cell representation in the anterodorsal thalamus after lesions of the retrosplenial cortex. J Neurosci 30:5289-5302. CrossRef Medline

Destrieux C, Fischl B, Dale A, Halgren E (2010) Automatic parcellation of human cortical gyri and sulci using standard anatomical nomenclature. Neuroimage 53:1-15. CrossRef Medline

Dhindsa K, Drobinin V, King J, Hall GB, Burgess N, Becker S (2014) Examining the role of the temporo-parietal network in memory, imagery, and viewpoint transformations. Front Hum Neurosci 8:709. CrossRef

Ekstrom A, Suthana N, Millett D, Fried I, Bookheimer S (2009) Correlation between BOLD fMRI and theta-band local field potentials in the human hippocampal area. J Neurophysiol 101:2668-2678. Medline

Etienne AS, Jeffery KJ (2004) Path integration in mammals. Hippocampus 14:180-192. CrossRef Medline

Gamberini M, Bakola S, Passarelli L, Burman KJ, Rosa MG, Fattori P, Galletti C (2016) Thalamic projections to visual and visuomotor areas (V6 and V6A) in the Rostral Bank of the parieto-occipital sulcus of the Macaque. Brain Struct Funct 221:1573-1589. CrossRef Medline

Goodridge JP, Taube JS (1997) Interaction between the postsubiculum and 
anterior thalamus in the generation of head direction cell activity. J Neurosci 17:9315-9330. Medline

Greve DN, Fischl B (2009) Accurate and robust brain image alignment using boundary-based registration. Neuroimage 48:63-72. CrossRef Medline

Grill-Spector K, Henson R, Martin A (2006) Repetition and the brain: neural models of stimulus-specific effects. Trends Cogn Sci 10:14-23. CrossRef Medline

Han X, Becker S (2014) One spatial map or many? Spatial coding of connected environments. J Exp Psychol Learn Mem Cogn 40:511-531. CrossRef

Ino T, Inoue Y, Kage M, Hirose S, Kimura T, Fukuyama H (2002) Mental navigation in humans is processed in the anterior bank of the parietooccipital sulcus. Neurosci Lett 322:182-186. CrossRef Medline

Jakab A, Blanc R, Berényi EL, Székely G (2012) Generation of individualized thalamus target maps by using statistical shape models and thalamocortical tractography. AJNR. Am J Neuroradiol 33:2110-2116. CrossRef Medline

Jenkinson M, Bannister P, Brady M, Smith S (2002) Improved optimization for the robust and accurate linear registration and motion correction of brain images. Neuroimage 17:825-841. CrossRef Medline

Johnson JD, Rugg MD (2007) Recollection and the reinstatement of encoding-related cortical activity. Cereb Cortex 17:2507-2515. CrossRef Medline

Knight R, Piette CE, Page H, Walters D, Marozzi E, Nardini M, Stringer S, Jeffery KJ (2014) Weighted cue integration in the rodent head direction system. Philos Trans R Soc Lond B Biol Sci 369:20120512. CrossRef Medline

Krauth A, Blanc R, Poveda A, Jeanmonod D, Morel A, Székely G (2010) A mean three-dimensional atlas of the human thalamus: generation from multiple histological data. Neuroimage 49:2053-2062. CrossRef Medline

Kremers NA, Deuker L, Kranz TA, Oehrn C, Fell J, Axmacher N (2014) Hippocampal control of repetition effects for associative stimuli. Hippocampus 24:892-902. CrossRef Medline

Maguire EA (2001) The retrosplenial contribution to human navigation: a review of lesion and neuroimaging findings. Scand J Psychol 42:225-238. CrossRef Medline

Marchette SA, Vass LK, Ryan J, Epstein RA (2014) Anchoring the neural compass: coding of local spatial reference frames in human medial parietal lobe. Nat Neurosci 17:1598-1606. CrossRef Medline

Meilinger T, Riecke BE, Bülthoff HH (2014) Local and global reference frames for environmental spaces. Q J Exp Psychol 67:542-569. CrossRef Medline

Montello DR, Pick HL (1993) Integrating knowledge of vertically aligned large-scale spaces. Env Behav 25:457-484. CrossRef

Morel A (2007) Stereotactic atlas of the human thalamus and basal ganglia. New York: Informa Healthcare USA.

Norman KA, O'Reilly RC (2003) Modeling hippocampal and neocortical contributions to recognition memory: a complementary-learningsystems approach. Psychol Rev 110:611-646. CrossRef Medline

Patenaude B, Smith SM, Kennedy DN, Jenkinson M (2011) A Bayesian model of shape and appearance for subcortical brain segmentation. Neuroimage 56:907-922. CrossRef Medline

Shinder ME, Taube JS (2014) Self-motion improves head direction cell tuning. J Neurophysiol 111:2479-2492. CrossRef Medline

Smith SM (2002) Fast robust automated brain extraction. Hum Brain Mapp 17:143-155. CrossRef Medline

Smith SM, Nichols TE (2009) Threshold-free cluster enhancement: addressing problems of smoothing, threshold dependence and localisation in cluster inference. Neuroimage 44:83-98. CrossRef Medline
Snyder LH, Grieve KL, Brotchie P, Andersen RA (1998) Separate body- and world-referenced representations of visual space in parietal cortex. Nature 394:887-891. CrossRef Medline

Souman JL, Frissen I, Sreenivasa MN, Ernst MO (2009) Walking straight into circles. Curr Biol 19:1538-1542. CrossRef Medline

Squire LR, Stark CE, Clark RE (2004) The medial temporal lobe. Annu Rev Neurosci 27:279-306. CrossRef Medline

Stackman RW, Taube JS (1997) Firing properties of head direction cells in the rat anterior thalamic nucleus: dependence on vestibular input. J Neurosci 17:4349-4358. Medline

Taube JS (1995) Head direction cells recorded in the anterior thalamic nuclei of freely moving rats. J Neurosci 15:70-86. Medline

Taube JS (2007) The head direction signal: origins and sensory-motor integration. Annu Rev Neurosci 30:181-207. CrossRef Medline

Taube JS, Muller RU, Ranck JB Jr (1990a) Head-direction cells recorded from the postsubiculum in freely moving rats: I. Description and quantitative analysis. J Neurosci 10:420-435. Medline

Taube JS, Muller RU, Ranck JB Jr (1990b) Head-direction cells recorded from the postsubiculum in freely moving rats: II. Effects of environmental manipulations. J Neurosci 10:436-447. Medline

Taube JS, Valerio S, Yoder RM (2013) Is navigation in virtual reality with FMRI really navigation? J Cogn Neurosci 25:1008-1019. CrossRef Medline

Tulving E, Thomson DM (1973) Encoding specificity and retrieval processes in episodic memory. Psychol Rev 80:352-373. CrossRef

Vass LK, Epstein RA (2013) Abstract representations of location and facing direction in the human brain. J Neurosci 33:6133-6142. CrossRef Medline

Watrous AJ, Ekstrom AD (2014) The spectro-contextual encoding and retrieval theory of episodic memory. Front Hum Neurosci 8:75. CrossRef Medline

Wilber AA, Clark BJ, Forster TC, Tatsuno M, McNaughton BL (2014) Interaction of egocentric and world-centered reference frames in the rat posterior parietal cortex. J Neurosci 34:5431-5446. CrossRef Medline

Wimber M, Maa $\beta$ A, Staudigl T, Richardson-Klavehn A, Hanslmayr S (2012) Rapid memory reactivation revealed by oscillatory entrainment. Curr Biol 22:1482-1486. CrossRef Medline

Winkler AM, Ridgway GR, Webster MA, Smith SM, Nichols TE (2014) Permutation inference for the general linear model. Neuroimage 92:381-397. CrossRef Medline

Wobbrock JO, Findlater L, Gergle D, Higgins JJ (2011) The aligned rank transform for nonparametric factorial analyses using only ANOVA procedures. Proceedings of the ACM Conference on Human Factors in Computing Systems (CHI '11). Vancouver, British Columbia (May 7-12, 2011), pp. 143-146. New York: ACM.

Wolbers T, Hegarty M, Büchel C, Loomis JM (2008) Spatial updating: how the brain keeps track of changing object locations during observer motion. Nat Neurosci 11:1223-1230. CrossRef Medline

Wolbers T, Wiener JM (2014) Challenges for identifying the neural mechanisms that support spatial navigation: the impact of spatial scale. Front Hum Neurosci 8:571. CrossRef Medline

Yoder RM, Taube JS (2014) The vestibular contribution to the head direction signal and navigation. Front Integr Neurosci 8:32. CrossRef Medline

Zhang H, Copara M, Ekstrom AD (2012) Differential recruitment of brain networks following route and cartographic map learning of spatial environments. PloS One 7:e44886. CrossRef Medline 